\title{
Medical Image of the Week: Typical Pulmonary CT Findings Following Radiotherapy
}

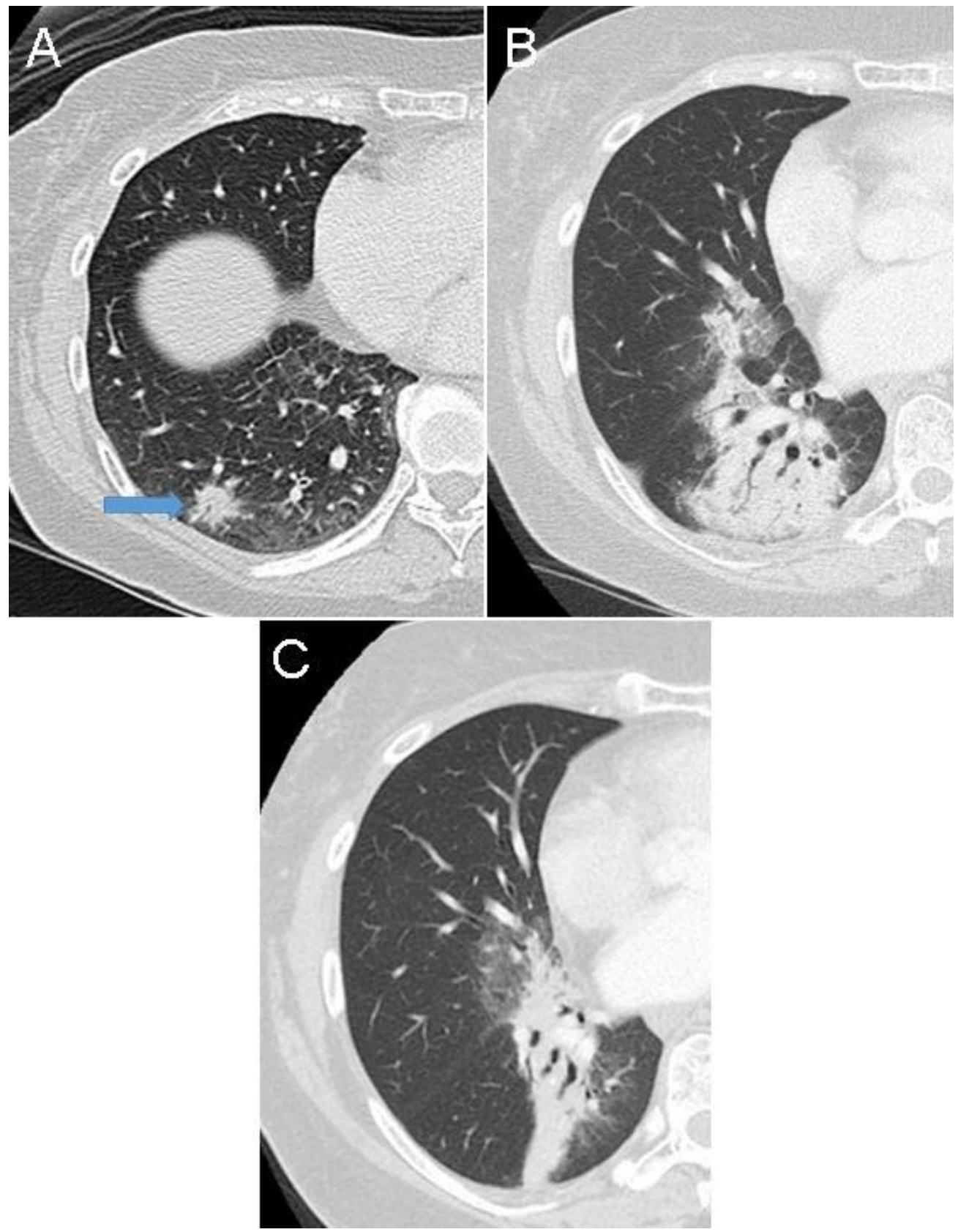

Figure 1. Panel A: CT chest, lung windows, demonstrating a spiculated nodule, biopsy proven adenocarcinoma in the right lower lobe (arrow). Panel B: Eight months post stereotactic radiation therapy, there has been development of focal consolidation, with air bronchograms, involving the right middle and lower lobes. Notice the volumetric appearance. The primary malignancy is no longer identified as such. Panel C: Thirteen months later the consolidation has evolved into an area of volume loss, containing bronchiectasis, and sharp contours as a result of organized fibrosis. 
Radiation-induced lung disease (RILD) commonly develops in patients treated with radiation for intrathoracic and chest wall malignancies.

There are two distinct radiographic patterns:

1. Radiation pneumonitis which occurs within 4-12 weeks after completion of therapy, and is characterized by development ground-glass opacities and/or consolidation in and around the treated lesion. A somewhat nodular or patchy appearance may occur. Typically, the affected tissue conforms to the radiation ports and may cross fissures/lobes. There may be milder similar changes in the contralateral lung.

2. A chronic phase, known as radiation fibrosis, is noticeable about 6-12 months post treatment and may progress up to 2 years, after which the findings tend to stabilize. In this stage, the areas of consolidation undergo volume loss, architectural distortion and may contain traction bronchiectasis. Linear and band scarring may also be seen. In this phase, sharper demarcation between normal and irradiated lung parenchyma is commonly seen.

Special attention to the typical radiological characteristics and timeline, in most cases allows to distinguish RILD from potential superimposed infection, subacute inflammatory diseases, locally recurrent neoplasm and radiation-induced neoplasms.

Andrew Erickson MS IV ${ }^{1}$, Berndt Schmidt MD², Veronica Arteaga MD², Diana Palacio $\mathrm{MD}^{2}$

${ }^{1}$ Midwestern University - Arizona College of Osteopathic Medicine

${ }^{2}$ Division of Thoracic Radiology, Department of Medical Imaging. University of Arizona, Tucson (AZ)

\section{Reference}

1. Choi YW, Munden RF, Erasmus JJ, Joo Park K, Chung WK, Jeon SC, Park CK. Effects of radiation therapy on the lung: radiologic appearances and differential diagnosis. Radiographics. 2004 Jul;24(4):985-97. [CrossRef] [PubMed] 